\title{
Dementia in rural South Africa: A pressing need for epidemiological studies
}

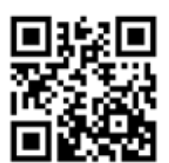

Dementia is a growing public health concern globally, with total estimated worldwide costs per year at USD604 billion in 2010. ${ }^{[1]}$ With older age being a major risk factor for dementia, the increasing numbers of older adults worldwide will increase demand for services to diagnose, treat and care for people with dementia (PWD). Little is known about the prevalence of dementia or its impact on older adults living in low- and middle-income countries (LMICs) in Africa, including South Africa (SA). Furthermore, there has been little research into the aetiology and risk factors in LMICs. The need for studies to investigate these factors in SA is therefore critical.

Primary dementia is a non-communicable disease (NCD) or syndrome, usually of a chronic or progressive nature, caused by neurodegeneration in the brain that affects a range of cognitive domains including memory, language, orientation and executive functions. As a result, behaviour, mood and the ability to perform everyday activities are affected. Although dementia mainly affects older people, it is not a normal part of ageing. Alzheimer's disease (AD) is the most common form of dementia in older adults and possibly contributes to $60-70 \%$ of cases. ${ }^{[2]}$ Other major varieties include vascular dementia, dementia with Lewy bodies and frontotemporal dementia. In SA, disorders associated with neurodegeneration such as traumatic brain injury, alcohol dependence and HIV infection are affecting increasing numbers of older adults. There is also a growing burden of disease from NCDs such as diabetes, heart disease and obesity resulting from increasingly unhealthy lifestyles and diet, which contribute to dementia risk. ${ }^{[3]}$ Dementia causes a loss of independence and is overwhelming for individuals living with the condition, their carers and their families. Comorbidities and frailty become increasingly common as dementia progresses, requiring more health, social and home/community care services.

\section{Worldwide prevalence}

Recent estimates are that there are approximately 44 million PWD worldwide, with $60 \%$ of these living in LMICs. This percentage is predicted to rise to $71 \%$ by $2050,{ }^{[4]}$ with expected increases between $134 \%$ and $146 \%$ in Latin America, North Africa and the Middle East. Projected increases for sub-Saharan Africa are between 70\% and $90 \%$. This relatively modest increase is consistent with limited demographic ageing owing to high child and adult mortality and the HIV epidemic. ${ }^{[2]}$ Estimated age-standardised dementia prevalences for individuals aged $>60$ years have been reported for 21 Global Burden of Disease regions, ${ }^{[1]}$ with a four-fold variation in prevalence from $2.1 \%$ to $8.5 \%$. The $10 / 66$ Dementia Research Group conducted a multicentre study in ten LMICs that included Nigeria but not SA. The dementia prevalence using Diagnostic and Statistical Manual of Mental Disorders, 4th edition (DSM-IV) criteria was 3.9\% for persons aged over 60 years. ${ }^{[5]}$ With the $10 / 66$ screening algorithm, prevalences were higher, ranging from $5.6 \%$ to $11.7 \%$, being lowest in rural China and India and highest in Cuba. The difference in prevalence with different diagnostic criteria was reportedly due to lack of sensitivity of the DSM-IV for less severe cases. Cases of dementia generally progress in severity over time, which calls for earlier detection.

Research on dementia conducted in sub-Saharan Africa indicates a higher prevalence in urban v. rural areas. The prevalence ranges from $2-3.7 \%$ in rural Nigeria and Benin to $6-7.6 \%$ in cities in
Central Africa. ${ }^{[6]}$ The differing prevalence ranges may reflect the methods employed in the studies, or differences in genetic, familial, environmental, cultural or educational factors in the regions studied. $\mathrm{AD}$ risk due to the ApoE4 allele is weaker for Yoruba Nigerians than for African Americans, ${ }^{[7]}$ and it is not known how genetic risk varies among other African populations. Moreover, milder dementia may be underdetected in LMICs because of low awareness, the high levels of support routinely provided to older people, and reluctance to report social and occupational failings to outsiders. These factors all contribute to difficulties in establishing a diagnosis.

\section{Dementia prevalence in SA}

There is a paucity of published research on the prevalence of dementia in SA. ${ }^{[8]}$ A survey of individuals aged $>60$ years in residential homes found $7.9 \%$ with dementia; this was not, however, a population prevalence study. The University of the Free State, with the 10/66 Group, investigated dementia in an urban black community and reported a higher than expected preliminary prevalence of approximately $6 \%$ in a small sample of 200 older persons. ${ }^{[9]}$

\section{Prevalence of HIV-associated dementia}

There is potentially a growing epidemic of dementia in older South Africans, especially among those with HIV infection. The prevalence of HIV-associated dementia (HAD) is $15-30 \%$ in untreated populations with late-stage disease, presenting with neurocognitive impairments, emotional disturbances, and motor dysfunction. ${ }^{[10]}$ The prevalence in individuals receiving highly active antiretroviral therapy (HAART) is $10 \%$, with an annual incidence of $1 \%$. The prevalence of less severe forms of HIV-associated neurocognitive disorder (HAND) is $20-30 \% \cdot{ }^{[11]}$ Prevalences are higher among people accessing HIV care in sub-Saharan African countries with a high HIV seroprevalence. Prevalences of $42 \%$ for HAND and $25 \%$ for HAD were reported among individuals starting HAART in primary care centres in Cape Town, ${ }^{[12]}$ with all study participants being in later stages of HIV infection. Cognitive disorder in HIV is particularly important in view of associations with poor adherence to HAART, faster disease progression and mortality. ${ }^{[12]}$ It is difficult to quantify the impact of numbers of people with dementia and of the age distribution of dementia cases in the region. Given an adult HIV seroprevalence of $15-25 \%$ in southern African countries, it is conceivable that most patients with dementia in the region are likely to have HAD. ${ }^{[10]}$

\section{Services for PWD in SA}

It is anticipated that by 2030 the proportion of the SA population aged $>60$ years will have increased to $11 \%,{ }^{[13]}$ with a concomitant rise in cases of dementia. However, the level of awareness and preparedness for the detection and costs of dementia care in the country is low, particularly in rural areas.

In 2011, SA had fewer than ten geriatricians and fewer than five specialists in old-age psychiatry for a population of 3.8 million persons aged $>60$ years. ${ }^{[14]}$ Basic curricula for training of health professionals in screening and diagnosis of dementia, and of social workers and other practitioners in management and care options for PWD, are limited. Dementia is often unrecognised by primary care practitioners, with signs and symptoms often ascribed simply 
to old age. As in many LMICs, primary level healthcare concentrates on treatable diseases. Health and social services often fail to meet the needs of older persons, especially those with mental health problems and dementia. ${ }^{[15]}$ In rural areas, the burden of HIV cases on the health system results in limited access to good treatment for PWD. ${ }^{[1]}$ Dementia drugs to improve cognitive function and treat underlying depression and/or behavioural disorders are expensive and unavailable on the Essential Drug List, and are therefore beyond the reach of the majority of older persons.

A great need for family support and care services for problems encountered with daily living and behaviour has been identified. A study in Cape Town found that $79 \%$ of a memory clinic's clients were being cared for by family members, some of whom had given up their jobs to do so. ${ }^{[16]}$ Caregiver stress is highlighted as an important factor in dementia care in LMICs, even in traditional cultures where family care is regarded as the norm. ${ }^{[15]}$ There are problems with access to care for older persons in urban areas, but the situation is worse in rural areas, where more than $40 \%$ of the SA population live and where service provision is fragmented and often severely underresourced. If dementia and its associated behavioural symptoms are poorly understood in rural communities, there is the risk that individuals with dementia may be stigmatised, with their symptoms ascribed to witchcraft, ostracised and subject to abuse. The policy on ageing in SA promotes deinstitutionalisation and limits statesubsidised residential care, so that no more than $2 \%$ of frail older persons who are in need of 24-hour nursing care are catered for. ${ }^{[17]}$ The national dementia associations, Alzheimer's SA and Dementia $\mathrm{SA}$, both non-governmental organisations (NGOs), do provide informal education and support for carers and pursue activist roles to improve both policy and services. Similarly, a few other NGOs, including religious groups, provide services for older people.

The paucity of epidemiological data in SA makes it difficult to advocate for improved services or prioritisation of funding for dementia studies. Research on prevalence and risk factors pertinent to the local population is needed to estimate the public health burden of dementia. Increased knowledge about dementia will enable earlier detection and diagnosis of this NCD. Thereafter, healthcare, interventions and support can be implemented and improved. This is particularly relevant at the present time in SA, as the government plans to introduce a National Health Service with National Health Insurance, and it would be timely to ensure that services for older persons are incorporated.

The following are needed now to address dementia in SA:

- Robust prevalence data using culture-fair screening tests

- Earlier diagnosis and intervention options

- Destigmatisation and increased awareness

- Increased preparedness and service provision

- Integration of older persons' needs and rights into national health policies across all sectors, e.g. health and justice, adequate protection of vulnerable PWD from abuse and neglect

- Prioritised research funding

- Education at all levels, including communities, to better manage PWD and enable them to live meaningful and purposeful lives

- Training and skills development for healthcare providers
- Career advancement for professionals in geriatric medicine and related disciplines.

\section{Celeste A de Jager}

Divisions of Geriatric Medicine and Neurology, Department of Medicine, Faculty of Health Sciences, University of Cape Town, South Africa

\section{John A Joska}

Division of Neuropsychiatry, Department of Psychiatry and Mental Health, Faculty of Health Sciences, University of Cape Town, South Africa

\section{Margaret Hoffman}

School of Public Health and Family Medicine, Faculty of Health

Sciences, University of Cape Town, South Africa

Karen E Borochowitz

Dementia SA, Cape Town, South Africa

\section{Marc I Combrinck}

Divisions of Geriatric Medicine and Neurology, Department of Medicine, Faculty of Health Sciences, University of Cape Town, South Africa

\section{Corresponding author: C A de Jager (celeste.dejager@uct.ac.za)}

1. World Health Organization. Dementia: A Public Health Priority. Geneva: WHO, 2012. http:// www.who.int/mental_health/publications/dementia_report_2012/en/ (accessed 31 January 2015).

2. Alzheimer's Association. Alzheimer's disease facts and figures. Alzheimers Dement 2012;8(2):131-168. [http://dx.doi.org/10.1016/j.jalz.2012.02.001]

3. Mayosi BM, Flisher AJ, Lalloo UG, Sitas F, Tollman SM, Bradshaw D. The burden of noncommunicable diseases in South Africa. Lancet 2009;374(9693):934-947. [http://dx.doi.org/10.1016/ S0140-6736(09)61087-4]

4. Ferri $\mathrm{CP}$, Prince $\mathrm{M}$, Brayne $\mathrm{C}$, et al; Alzheimer's Disease International. Global prevalence of dementia: 4. Ferri CP, Prince M, Brayne C, et al; Alzheimer's Disease International. Global prevalence of dementia:
A Delphi consensus study. Lancet 2005;366(9503):2112-2117. [http://dx.doi.org/10.1016/\$01406736(05)67889-0]

5. Prince M, Acosta D, Chui H, et al. Dementia diagnosis in developing countries: A cross-cultural validation study. Lancet 2003;361(9361):909-917. [http://dx.doi.org/10.1016/S0140-6736(03)12772-9] 6. Guerchet M, Mouanga AM, M'belesso P, et al. Factors associated with dementia among elderly people living in two cities in Central Africa: The EDAC multicentre study. J Alzheimers Dis 2012;29(1):15-24. [http://dx.doi.org/10.3233/JAD-2011-111364]

7. Hendrie HC, Murrell J, Baiyewu O, et al. APOE $\varepsilon 4$ and the risk for Alzheimer disease and cognitive decline in African Americans and Yoruba. Int Psychogeriatr 2014;26(6):977-985. [http://dx.doi. org/10.1017/S1041610214000167]

8. Olayinka OO, Mbuyi NN. Epidemiology of dementia among the elderly in sub-Saharan Africa. Int J Alzheimers Dis 2014;2014:195750. [http://dx.doi.org/10.1155/2014/195750]

9. Radebe M. Media release, Media Liaison. http://www.ufs.ac.za/templates/archive. aspx?news=1871\&cat=1 (accessed 31 January 2015).

10. Grant I. Neurocognitive disturbances in HIV. Int Rev Psychiatry 2008;20(1):33-47. [http://dx.doi. org/10.1080/09540260701877894]
or

11. Valcour VG, Shikuma CM, Walters MR, et al. Cognitive impairment in older HIV-1-seropositive individuals: Prevalence and potential mechanisms. AIDS 2004;18(Suppl 1):S79-S86.

12. Joska JA, Westgarth-Taylor J, Myer L, et al. Characterization of HIV-associated neurocognitive disorders among individuals starting antiretroviral therapy in South Africa. AIDS Behav 2011;15(6):1197-1203. [http://dx.doi.org/10.1007/s10461-010-9744-6]

13. United Nations, Department of Economic and Social Affairs, Population Division. World Mortality Report. New York: UN, 2013.

14. Kalula S, Petros G. Responses to dementia in less developed countries with a focus on South Africa. Global Aging 2011;7(1):31-40.

15. Patel V, Prince M. Ageing and mental health in a developing country: Who cares? Qualitative studies from Goa, India. Psychol Med 2001;31(1):29-38.

16. Kalula SZ, Ferreira M, Thomas KGF, de Villiers L, Joska JA, Geffen LN. Profile and management of patients at a memory clinic. S Afr Med J 2010;100(7):449-451.

17. Follentine S. Ageing in South Africa: An overview. Bold 2006;16(4):7-16.

S Afr Med J 2015;105(3):189-190. DOI:10.7196/SAMJ.8904 